\title{
DEPTH SELECTIVE LASER SCRIBING OF THIN-FILM SILICON SOLAR CELLS ON FOIL
}

\author{
Paper M1106
}

\author{
Laurie Wipliez $^{1^{*}}$, Jochen Löffler ${ }^{1}$, Martijn de Keijzer ${ }^{1}$, Wim Soppe ${ }^{1}$, Thomas Sebastien ${ }^{1}$ \\ Aart Schoonderbeek ${ }^{2}$, Oliver Haupt ${ }^{2}$, Uwe Stute ${ }^{2}$ \\ ${ }^{1}$ Energy Research Centre of The Netherlands, P.O. Box 1, 1755 ZG Petten, The Netherlands. \\ ${ }^{2}$ Laser Zentrum Hannover e.V., Hollerithallee 8, D-30419 Hannover, Germany \\ "Corresponding author, phone +31 (224) 56 4420, e-mail: wipliez@ecn.nl
}

\begin{abstract}
Roll-to-roll production of thin-film photovoltaic (PV) solar cells and modules is expected to decrease substantially the manufacturing costs, and thus enable a breakthrough in the price of solar electricity per kWh. The roll-to-roll concept implies that the fabrication of these PV devices on flexible substrates is significantly different from the production of the glass based devices. This is especially valid for the monolithic series interconnection of thin-film silicon solar cells into modules, where the laser scribing step of thin-films on opaque foils requires depth selectivity. As adjusting the laser wavelength to the absorption profiles of the involved layers is not sufficient, we are investigating the ablation mechanisms leading to removal of the different layers of thin-film silicon solar cells.
\end{abstract}

In this paper, first results of laser scribes into working solar cells are reported using $1064 \mathrm{~nm}$ nanosecond pulsed lasers. Despite the apparent depth selectivity reported earlier for this type of laser, a reduced diode quality and/or shunting of the solar cells is observed. This is probably due to recast at the wall of the laser scribe either by molten material from the back contact or by re-crystallized silicon. Consequently, a broader wavelength/pulse duration matrix has been experimented to understand better the ablation processes of the individual layers, aiming at a reduction of damage of the $\mathrm{PV}$ devices due to the laser process.

Finally, laser scribes with reduced damage on working solar cells have been achieved with a near IR picosecond laser, leading to lower losses of the solar cell efficiency than with the $1064 \mathrm{~nm}$ nanosecond laser.

\section{Introduction}

The last few years, thin-film solar cells have received a growing interest as a cost effective technology for photovoltaic energy generation, in comparison with the more traditional crystalline silicon wafer-based technology. Roll-to-roll production of thin-film PV promises to reduce further the production costs. In order to fabricate thin-film modules, solar cells deposited on a same substrate must be insulated from each other and the back contact of one cell must be connected to the front contact of its neighbor. This is an essential process step in the realization of the modules, which is called "monolithic series interconnection" and offers a high cost reduction potential in manufacturing methods. For the interconnection, a commonly used process in industry is the laser structuring of individual layers constituting the solar cell. Recently, many production lines for thinfilm silicon PV modules based on glass substrates have become operational, and laser scribing tools and processes are available on the market. However, this interconnection process requires three separate vacuum deposition steps (for TCO, silicon layers, and rear contact, respectively), each one followed by a laser scribing step in atmosphere [1].

Therefore, an important decrease in production costs is expected when combining roll-to-roll production with the realization of the interconnection by one single process step of three depth selective laser scribes, after applying all layers. Today, in contrast to production lines based on glass substrates, no standard equipment is available for this one-step interconnection of thinfilm silicon solar cells and modules on opaque substrates. To overcome this limitation, ECN is currently developing the technology for the production of low-cost and high efficiency tandem solar cells based on microcrystalline and amorphous silicon on steel foil substrates [2], including laser scribing for monolithic series interconnection.

The ECN device consists of an insulating barrier layer on top of a conducting steel foil substrate. On top of this barrier layer, a sputtered back contact is applied, followed by PECVD of the active silicon layers and the front TCO sputtering. After all solar cell layers are deposited, the three depth selective laser scribes P1, P2 and $\mathrm{P} 3$ are realized in one single step on this full stack, as can be seen in Figure 1. 


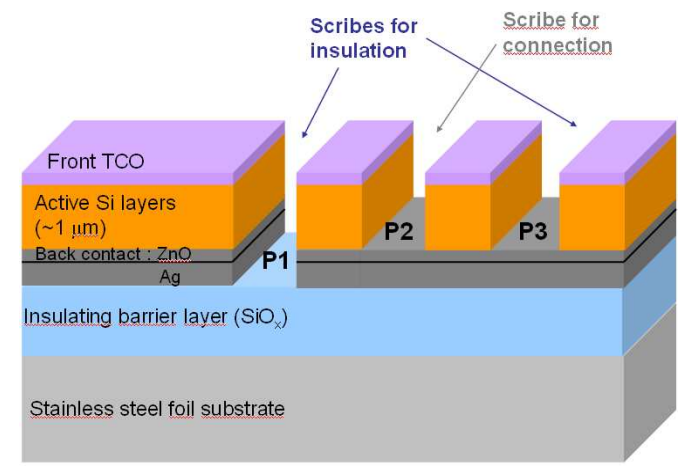

Figure 1: Three depth selective laser scribes for thinfilm silicon PV Module concept on electrically insulated steel foil substrate.

The P1 scribe has the function to separate electrically the cells from each other. Thus, all the layers of the cell have to be ablated, including the back contact. The main challenge is to have no remaining bridges of back contact in the scribe, while the insulating layer should be unaffected. This scribe will be subsequently filled by an insulating ink. The laser scribe $\mathrm{P} 2$ is necessary for the actual connection of the front contact of one cell to the back contact of the adjacent cell. P2 should remove all silicon layers, but leave the $\mathrm{ZnO} / \mathrm{Ag}$ back contact unaffected. This scribe will be then filled by an electrically conductive ink. The main function of the P3 scribe is to prevent lateral shunts of the ITO and silicon layers, and in principle, $\mathrm{P} 3$ can be obtained with the same process as P2. In this paper, all observations and discussions regarding P2 scribes are also valid for P3.

In [3], we presented results on depth selectivity studies with nanosecond pulsed lasers at different wavelengths on layer stacks resembling the final device. It was shown that for both P1 and P2 scribes, the process window for depth selectivity was limited when scribing with an UV nanosecond laser and a broader process window had been unexpectedly obtained with a near IR nanosecond laser.

Consequently, several laser pulse durations and wavelengths are experimented on the ECN full stack sample (as displayed in Figure 1), in order to assess the potential in depth selectivity of these laser sources. Before scribing lines on functional solar cells, the ablation mechanisms and ablation thresholds for the P1 and $\mathrm{P} 2$ processes are evaluated by scribing separated spots. These spots are studied with optical and confocal microscopy in order to calculate the different peak fluence ablation thresholds for each layer and to investigate the ablation processes.
We present in this paper the results and discussions on the ablation mechanisms when scribing the ECN full stack sample with near IR nanosecond and picosecond lasers. In addition, best laser scribing parameters are also tested in functional solar cells, evaluating the device performance before and after the laser step. In first instance, issues with short-circuiting of the solar cell have been encountered for near IR ns laser scribes, but this shunting issue could be minimized using shorter pulse duration. The ablation mechanisms due to other wavelengths and pulse durations are currently under investigation.

\section{Experimental Details}

\section{1) Laser Systems}

In this work, the ablation of the different individual layers constituting the thin-film silicon solar cells was characterized using nanosecond and picosecond laser pulses with a wavelength of $1064 \mathrm{~nm}$ (near IR). The lasers used were a Powerline 20E Series Diode Pumped Solid State Nd:YAG laser from Rofin Baasel with 25 nanosecond pulses, and a Lumera Staccato laser with 12 picosecond pulses. These lasers described in Table 1 have a beam with Gaussian power density distribution.

Table 1 Laser sources used for the experiments.

\begin{tabular}{|c|c|c|c|}
\hline Laser & $\begin{array}{c}\text { wavelength } \\
\lambda[\mathrm{nm}]\end{array}$ & $\begin{array}{c}\text { pulse } \\
\text { duration } \mathrm{t}_{\mathrm{p}}\end{array}$ & $\begin{array}{c}\text { calculated focus } \\
{\text { diameter }\left(1 / \mathrm{e}^{2}\right)[4]}_{[\mu \mathrm{m}]}\end{array}$ \\
\hline Rofin & 1064 & $25 \mathrm{~ns}$ & $101 \mu \mathrm{m}$ \\
\hline Lumera & 1064 & $12 \mathrm{ps}$ & $30 \mu \mathrm{m}$ \\
\hline
\end{tabular}

The lasers were equipped with galvo-head scanners to guide the laser over the substrates resulting in locally isolated spots, or when overlapping subsequent spots, in continuous lines. The output power was directly controlled by an external attenuator, and measured with a power meter at the exit of the scanner.

\section{2) Samples}

The investigated samples were fabricated by spraycoating of a thermally curing $\mathrm{SiO}_{\mathrm{x}}$ based sol-gel lacquer with blue color additives on $70 \mu \mathrm{m}$ thick stainless steel foil substrates, followed by magnetron sputtering of a silver layer and a $\mathrm{ZnO}$ :Al layer on top, respectively 100 and $80 \mathrm{~nm}$ thick. $400 \mathrm{~nm}$ of amorphous silicon layers were then deposited by PECVD. As front TCO, $80 \mathrm{~nm}$ of ITO has been applied by RF magnetron sputtering.

For the evaluation of the laser scribing effect on the IV-behavior of solar cells, a mask was employed to 
deposit the top TCO, in order to have $4 \times 4 \mathrm{~mm}^{2}$ small solar cells.

\section{3) Theory and Methodology}

In order to evaluate and understand the interaction between laser pulses and the ECN full stack sample, a starting point is the analysis of the spots with an optical microscope and the determination of the peak fluence ablation threshold, i.e. the amount of energy density needed for effective material removal as a function of pulse duration. The peak laser fluence $\phi_{0}$ (in $J / \mathrm{cm}^{2}$ ) is related with the applied pulse energy $E_{P}$ (in $J$ ) and the $1 / \mathrm{e}^{2}$-beam radius $\omega_{0}$ (in $\mu \mathrm{m}$ ) or the focal beam diameter $\mathrm{d}_{\mathrm{f}}\left(2 \omega_{0}\right.$, in $\left.\mu \mathrm{m}\right)$ :

$$
\phi_{0}=\frac{2 E_{P}}{\pi \omega_{0}^{2}}
$$

The applied pulse energy $E_{P}$ is calculated by dividing the average laser power $\mathrm{P}$ by the repetition rate.

A straightforward method to determine the threshold fluence for laser-induced bulk material modification has been introduced by J.M. Liu in 1982 [5], and it has been adopted by several other research groups to investigate thin-film removal by laser pulses $[6,7]$. Assuming a perfect Gaussian distribution with a focal beam diameter $d_{f}$ and peak fluence $\phi_{0}\left(\right.$ in $J / \mathrm{cm}^{2}$ ), the ablated crater will have a diameter $d_{a b l}($ in $\mu \mathrm{m}$ ) related to the peak fluence ablation threshold $\phi_{\text {th }}$ (in $\mathrm{J} / \mathrm{cm}^{2}$ ), following the equation (2):

$$
d_{a b l}^{2}=\frac{d_{f}^{2}}{2} \ln \left(\frac{\phi_{0}}{\phi_{t h}}\right)
$$

From the previous equation, it is possible to determine the $1 / \mathrm{e}^{2}$-beam diameter $\mathrm{d}_{\mathrm{f}}$ (in $\mu \mathrm{m}$ ) experimentally, from the slope of the graph representing the squared crater diameters $d_{a b l}$ versus the logarithm of the pulse energy $E_{P}$. Then, the laser pulse energy $E_{P}$ can be converted into peak fluence using equation (1), and a value for the ablation threshold peak fluence $\phi_{\text {th }}$ can be obtained from the plot of squared ablation diameters $\mathrm{d}_{\mathrm{abl}}{ }^{2}$ versus peak laser fluence $\phi_{0}$, by extrapolation of $\mathrm{d}_{\mathrm{abl}}^{2}$ back to zero.

In order to determine the different peak fluence ablation thresholds, for near IR lasers with two different pulse durations, we irradiated our sample with separated laser spots, varying the pulse energy $E_{P}$, and keeping the step size constant at $100 \mu \mathrm{m}$. Confocal microscopy was used to measure the ablation diameters, taking into account the depth selectivity (determined by optical microscopy images).

In a next step, the scanner speed was reduced in order to overlap the spots and scribe lines, aiming at best depth selectivity and cleanest scribes. Then, best laser parameters were chosen and tested on functional solar cells. The current-voltage curves of $4 \times 4 \mathrm{~mm}^{2}$ solar cells were first measured under a sun simulator, with one probe on the ITO surface and the other one on the back contact.

Then, in analogy with [1], the middle of these cells was scribed by a P1 or a P2 laser line. The laser scribe was $2 \mathrm{~mm}$ long and between 20 and $70 \mu \mathrm{m}$ wide, meaning that the dead area induced by the laser was negligible compared to the solar cell area. This allows us to investigate the effect of the laser scribe on the I-V behaviour of the solar cell, by re-measuring the I-V curve after scribing.

\section{Results and Discussion}

\section{1) Microscopy Studies}

The depth selectivity when scribing full amorphous silicon stack with near IR (1064 nm wavelength) nanosecond and picosecond lasers with a beam with Gaussian power density distribution has been investigated in first instance with optical microscopy. Figure 2 shows an overview with optical pictures of near IR nanosecond single laser spots on the ECN full stack sample, with increasing pulse energy from spot 1 to 6. Spots with different depths can be observed in this figure, increasing from spot 1 where the surface appears to be only slightly affected by the laser pulse, spot 2 with a bright area corresponding to the back contact layer visible in the center, spot 3 with a crack in the $\mathrm{ZnO} / \mathrm{Ag}$ layer, to spot 6 displaying the blue insulating barrier layer surrounded by a circle of back contact. Therefore, we define different ablation diameters for each different depth: an "affected area" diameter where the $400 \mathrm{~nm}$ thick amorphous silicon layer and $80 \mathrm{~nm}$ thick ITO layer appear to have only melted and cooled down (spot 1), a "P2 spot" diameter removing the silicon and ITO layers without ablating the rear contact (spots 2 and 3), and a "P1 spot" diameter where the ITO, the amorphous silicon and the $\mathrm{ZnO} / \mathrm{Ag}$ layers have been ablated displaying the surface of the blue barrier layer (spots 4, 5 and 6). The "affected area" diameters can be determined on these 6 different spots, the "P2 spot" diameters on spots 2 to 6 and "P1 spot" diameters on spots 4, 5 and 6. The depth selectivity of "P1" and "P2 spots" corresponds to the depth required for a P1 scribe and a P2 scribe, respectively. 


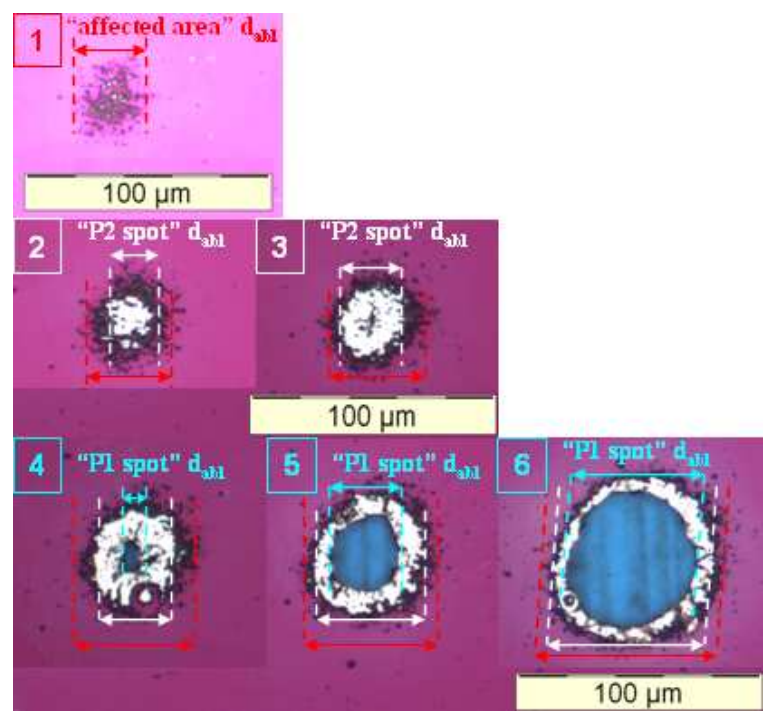

Figure 2: Optical microscope pictures of $1064 \mathrm{~nm}$ ns laser ablated spots ("affected area", "P2 spots" and "P1 spots") on the ECN amorphous silicon stack sample. The pulse energy increases from spot 1 to 6 . The white area on spots 2 and 3 corresponds to the back contact, and the blue area on spots 4,5 and 6 to the barrier layer.

To confirm and quantify the observations regarding the depth of the different spots, these craters have been analyzed with confocal microscopy, measuring their depth and the different diameters. Figure 3 shows the three dimensional height distribution, coded in false colors, of the spots 1,2 and 5, from confocal scans. When comparing the optical image of a low pulse energy laser spot (spot 1 in Figure 2) with its confocal 3D profile (see Figure 3), we can assert that the white points on the optical picture correspond to the back contact and the brown ones to peaks of molten and resolidified silicon, due to laser irradiation. Taking into account linear absorption processes in the different individual layers, neglecting the absorption in ITO, and considering that the amorphous silicon layer is highly transparent at a wavelength of $1064 \mathrm{~nm}$ [3], we assume that the laser beam is rather absorbed by the silver layer underneath the $\mathrm{ZnO}$, silicon and ITO. Therefore, the peaks visible on the 3D image from confocal measurement of spot 1 (Figure 3) are thought to illustrate the ablation mechanism with a pulse energy below the ablation threshold for a P2 spot: the laser irradiation heated and melted the back contact material through the silicon and ITO layers, but was not energetic enough to effectively remove the silicon and ITO. These two layers could have been expanded and pushed up by the back contact, and then froze with a shape of pillars, due to quick cooling down of the silicon.
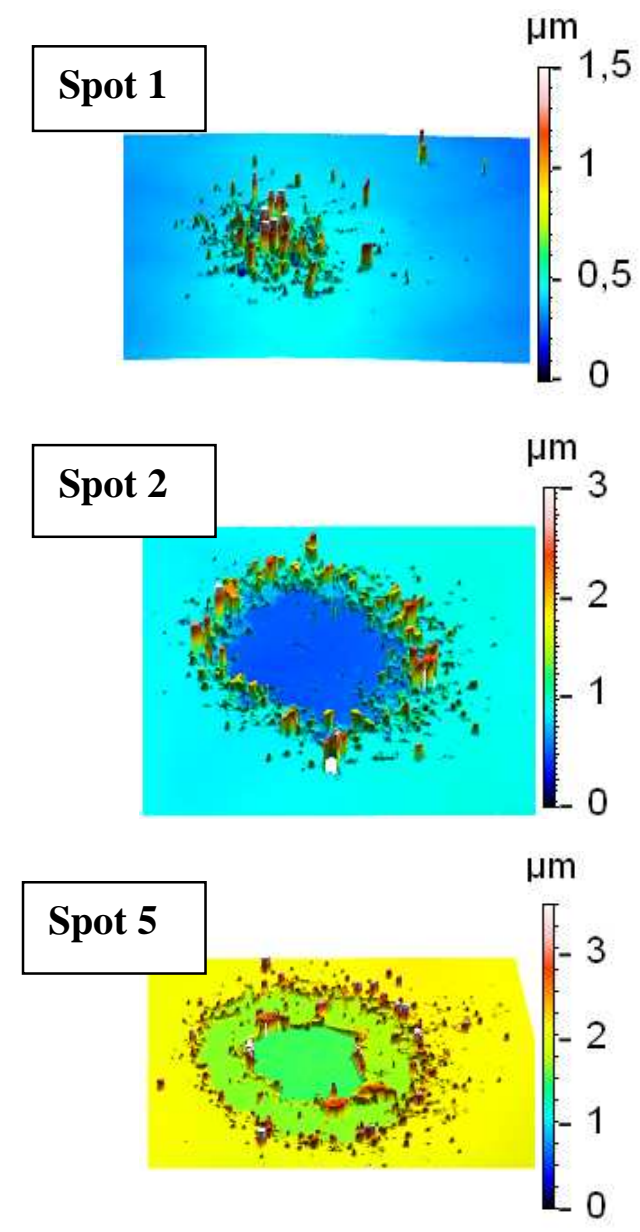

Figure 3: Three dimensional height distribution, from confocal scan of spots 1, 2 and 5 (number of the spots corresponding to Figure 2).

Employing enough pulse energy allows us to obtain P2 spots displaying the rear contact surface, as illustrated by spot 2 in Figure 2 and 3. The depth of the crater was measured to be $450 \mathrm{~nm}$, which corresponds approximately to the thickness of the silicon and ITO layers. The heating from the laser pulse could have expanded the back contact ( $\mathrm{ZnO}$ and Ag layers), which was then locally melting, evaporating and lifting off the silicon and ITO layers. It has to be mentioned that the influence of the $\mathrm{ZnO}$ layer in this process is still not fully clear, as it should be transparent to the laser wavelength. In addition, some pillars of silicon can be observed at the edges of the spot, showing that tails of the beam with Gaussian power density distribution were not energetic enough to remove the silicon. This 
observation confirms the ablation mechanism proposed above for a laser pulse with too low energy.

When the pulse energy is further increased, so is the depth of the crater resulting in a "P1 spot" which is approximately $600 \mathrm{~nm}$ deep, with the blue $\mathrm{SiO}_{\mathrm{x}}$ barrier layer visible at the center of the spot (see spot 5 in Figure 2 and 3). On the 3D image, the rear contact has been ablated in the middle of the spot, but some flakes of this layer are still connected to its surrounding. This indicates a more explosive ablation behavior of the rear contact, and it suggests that the $\mathrm{ZnO}$ and $\mathrm{Ag}$ layers could have been broken and opened by vapor pressure developed at the interface between the silver and the barrier layer. Heating and expansion of the $100 \mathrm{~nm}$ thin metal layer could also lead to removal of the rear contact (which includes $80 \mathrm{~nm}$ of $\mathrm{ZnO}$ on top of the silver layer) from the barrier layer. In order to have a better understanding of this process, single near IR ns laser pulses have been applied on samples without silicon and ITO layers, but only with back contact ( $\mathrm{ZnO}$ and $\mathrm{Ag}$ layers) sputtered on top of the barrier layer and steel substrate. Optical and confocal studies showed that some flakes and cracks were present at the edges of the spots, especially at pulse energy close to the ablation threshold, meaning that it is probably a high pressure ablation process.

Summarizing the results from the microscopy studies, it is expected that the investigated near IR ns laser ablation of amorphous silicon and ITO layers, necessary for a P2 scribe, results from heating of the metal from the rear contact underneath, which is then expanding and pushing up the silicon and ITO. The ablation of the $\mathrm{ZnO} / \mathrm{Ag}$ layer (for a $\mathrm{P} 1$ scribe) involves a more explosive process where the rear contact is broken by vapor pressure.

\section{2) Ablation thresholds}

Following the method described in [5], we employed the different ablation diameters measured with the confocal microscope, to determine the experimental focal beam diameter $d_{f}$, which was then used to convert the pulse energy $E_{P}$ into peak fluence $\phi_{0}$ (equation (1)). Figure 4 illustrates the ablated diameters $\mathrm{d}_{\mathrm{abl}}$ of "affected area", "P1" and "P2" spots on full stack sample versus peak laser fluence, of the near IR nanosecond laser. By extrapolating these fits to zero, we find the different peak fluence ablation thresholds, which are indicated in the graph (Figure 4).

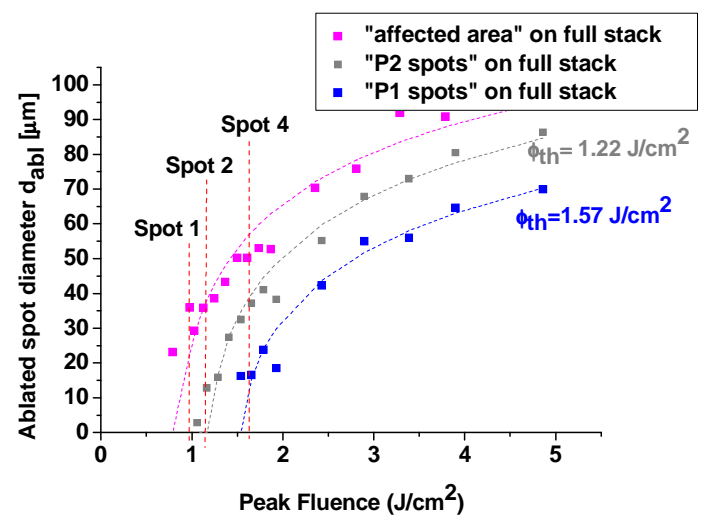

Figure 4: Ablation diameters $(\mu \mathrm{m})$ versus peak fluence

$\left(\mathrm{J} / \mathrm{cm}^{2}\right)$. The red lines indicate the peak fluences applied for spot 1 ("affected area"), spot 2 ("P2") and spot 4 ("P1"). The spots numbers correspond to Figure 2.

The peak fluence ablation threshold found for "P2 spots" is $1.22 \mathrm{~J} / \mathrm{cm}^{2}$ and the threshold for "P1 spots" is $1.57 \mathrm{~J} / \mathrm{cm}^{2}$, allowing a process window of $0.35 \mathrm{~J} / \mathrm{cm}^{2}$ between a P2 and a P1 spot. However, a reasonable opening size displaying 20 to $30 \mu \mathrm{m}$ of back contact is needed for a stable "P2 scribe" process, thus it reduces its process window. Indeed, the peak fluence needed for a larger opening displaying back contact increases with the size of the spot diameter, and is getting closer to the ablation threshold of a "P1" spot. Additionally, the Gaussian profile is another issue, considering that it is probably difficult to choose a pulse energy where the full Gaussian profile lies in the process window in terms of average fluence. Furthermore, the overlap needed to pass from single separated spots to lines may reduce the process window even further.

\section{3) Lines}

As discussed above, the expansion of the back contact could be responsible for the ablation mechanism of "P2 spots" on full stacks. In order to remove entire lines of silicon and ITO layers from the rear contact (P2) and of all three layers from the barrier layer (P1) at a laser wavelength of $1064 \mathrm{~nm}$, the depth has to be controlled by the laser parameters, adjusting the combination of pulse energy and overlap.

As the peak fluence ablation thresholds for P1 and P2 single laser spots with the near IR ns laser are already close to each other, it becomes difficult to obtain clean P2 scribes, without damage and holes in the back contact displaying the barrier layer, and without debris of silicon. Therefore, the process window for a clean removal of the silicon and ITO layers from the back contact is narrow, as can be observed in Figure 5, 
where only slightly too large or too little pulse energy or spot overlap results in either local removal of the back contact at the overlap areas, or silicon remaining in the scribe. The three lines displayed in this figure were scribed using an overlap of $60 \%$, and a fluence difference of $0.12 \mathrm{~J} / \mathrm{cm}^{2}$ between them. The optimum P2 scribe can be found in the middle of the picture, with minor damage to the rear contact, and with only little debris of silicon.

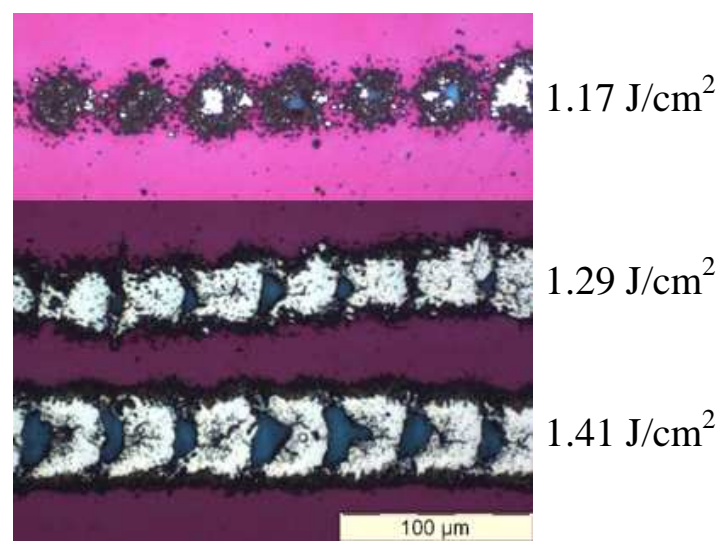

Figure 5: Three fluence levels to achieve a P2 scribe, at $60 \%$ overlap, with a $1064 \mathrm{~nm}$ ns pulsed laser. From top to bottom, the peak fluence was increased by steps of $0.12 \mathrm{~J} / \mathrm{cm}^{2}$.

On the other hand, there is a considerable process window for P1 scribes in terms of depth selectivity, with the near IR nanosecond laser. This is illustrated in Figure 6 where the fluence difference between the three scribes is the same as the one used between the P2 scribes in Figure 5. But they all meet optical requirements for $\mathrm{P} 1$, i.e. showing a clean surface of the barrier layer (blue area in the middle of the scribe) without any pieces of back contact connecting the opposite sides of the scribe.

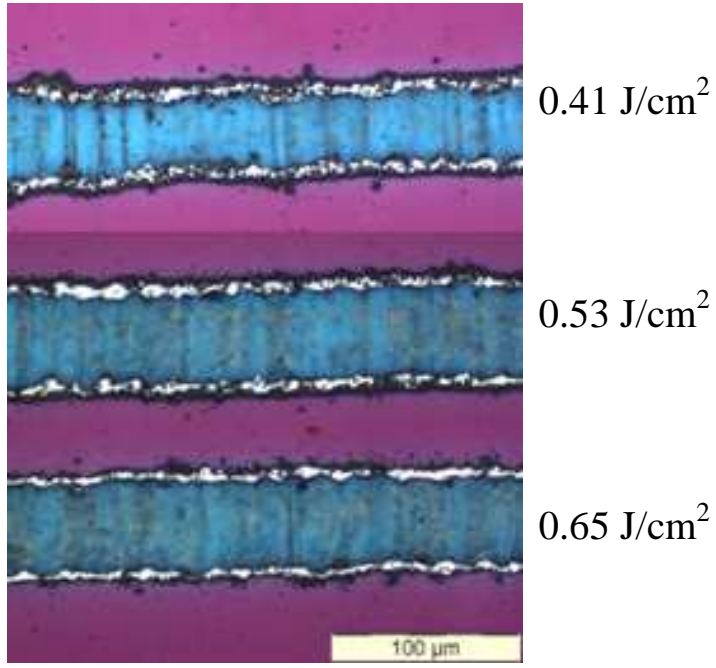

Figure 6: Three fluence levels to achieve a P1 laser scribe, at $92 \%$ overlap for the line on the top, and $84 \%$ overlap for the two scribes below, obtained with a $1064 \mathrm{~nm}$ ns pulsed laser. From top to bottom, the peak fluence was increased by steps of $0.12 \mathrm{~J} / \mathrm{cm}^{2}$.

\section{4) Thin-film silicon solar cells}

In the following part, the laser parameters used for the scribe in the middle in Figure 6 have been tested in functional solar cells. Despite the visually appealing scribe (see Figure 6), short-circuiting of the cell has been observed after scribing a P1 with the near IR nanosecond laser, which is illustrated by the I-V curves in Figure 7.

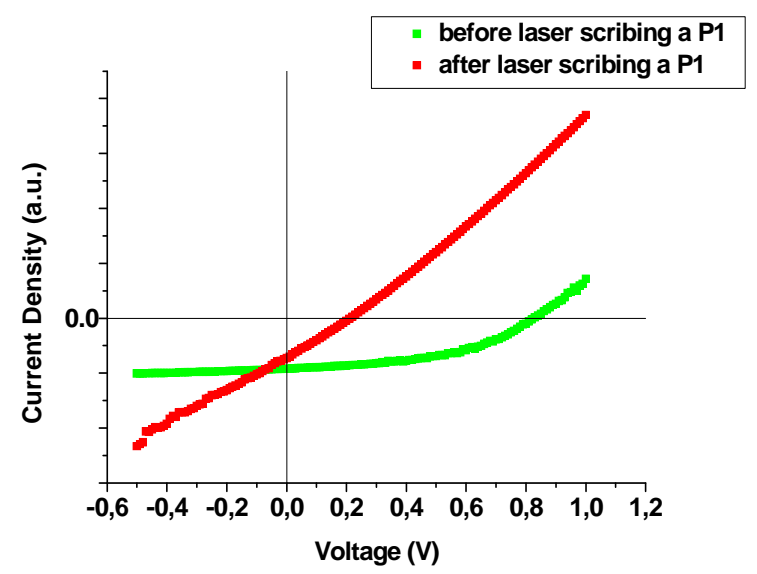

Figure 7: I-V curves under illumination before and after laser scribing a P1 line with the near IR ns laser.

This figure shows that laser scribing a P1 on a thinfilm silicon solar cell induced a significant drop in open circuit voltage $\left(\mathrm{V}_{\mathrm{OC}}\right)$, decreasing from around 
$0.8 \mathrm{~V}$ to $0.2 \mathrm{~V}$; and for a negative voltage, the current is not blocked anymore. Therefore, the solar cell is not a fully functional diode anymore, but became nearly an ohmic resistance, which is drastically limiting the efficiency of the cell after laser scribing.

In order to understand the origin of the shunting of the cells, confocal microscopy was used to study the P1 laser scribe. The confocal scan is illustrated in Figure 8 , where the shunting could be explained by recast and displaced back contact material projected onto the P1 scribe walls, due to the thermal character of the ablation by nanosecond laser pulses.

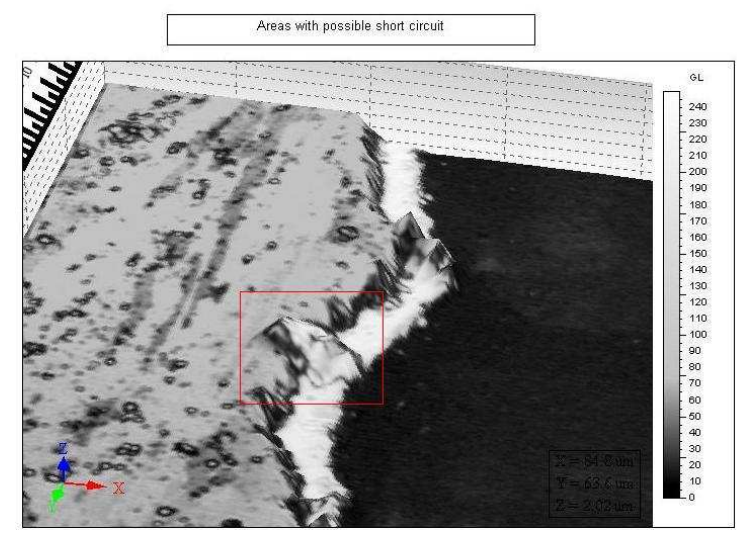

Figure 8: Possible shunt between back contact and front contact. Image from confocal microscopy.

For a similar process on CuInSe2-based solar cells, a connection between the front and rear contacts due to residues of back contact material projected onto the walls of the laser ablated scribe are identified by Hermann et al. [8], to be a cause of shunting of their photovoltaic device.

\section{5) Scribing with a near IR picosecond laser}

To find a solution for minimizing the degradation of the photo-electrical properties of the solar cell, the interaction of shorter near IR laser pulses (duration in picoseconds) with the ECN full stack sample has been investigated. The diameters of the craters obtained for different peak fluences are displayed in Figure 9. An ablation threshold of $1.3 \mathrm{~J} / \mathrm{cm}^{2}$ has been found for a "P2 spot" and $4.06 \mathrm{~J} / \mathrm{cm}^{2}$ for a "P1 spot", providing a process window of $2.76 \mathrm{~J} / \mathrm{cm}^{2}$ between a "P2" and a "P1" spot, which is eight times larger than with the nanosecond laser. This indicates a clear advantage of the near IR ps laser compared to the ns laser, allowing a much broader process window for a depth selective P2 scribe.

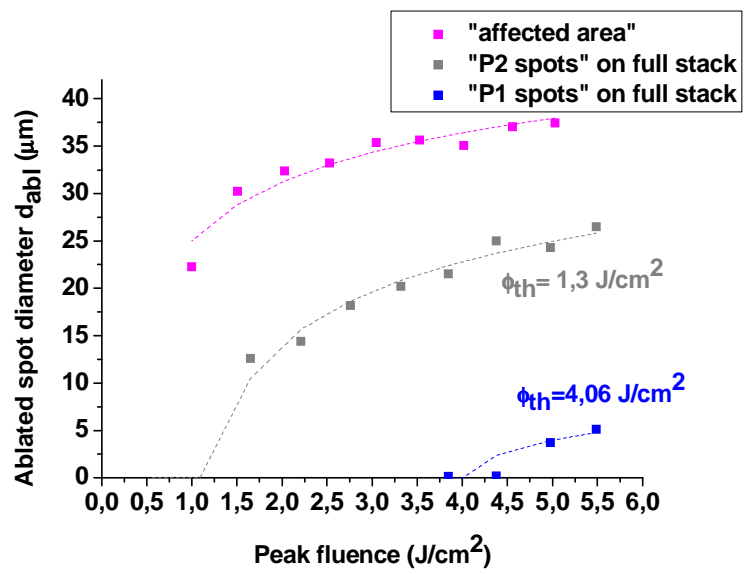

Figure 9: Ablation diameters $(\mu \mathrm{m})$ versus peak fluence $\left(\mathrm{J} / \mathrm{cm}^{2}\right)$, for near IR ps laser spots.

Indeed, comparing the graphs of ablated spot diameters versus peak fluence for near IR nanosecond laser spots with near IR picosecond spots, displayed in Figure 4 and 9 respectively, it can be easily observed that the process window between a "P1" and a "P2" spot is much larger using the ps laser, compared to the ns laser.

However, the rear contact of "P2 spots" obtained with the near IR ps laser was not as clean as the ones obtained with the ns laser. Indeed, a lot of debris of silicon was visible on the surface of the $\mathrm{ZnO}$ layer, and several cracks of the back contact could be observed on the optical and confocal images. An example of a "P2 spot" is given in Figure 10.
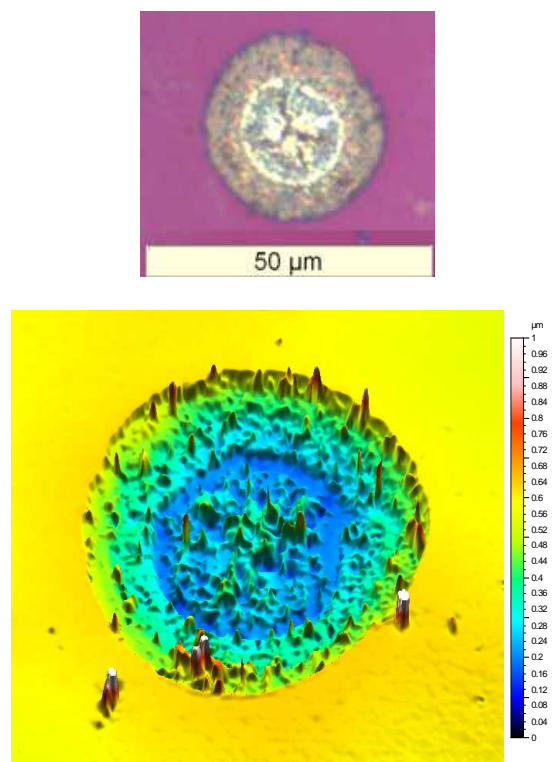

Figure 10: Optical and confocal images of a "P2 spot" scribed with the near IR ps laser. 
Unexpectedly, when overlapping the pulses, cleaner P2 lines, with a broad process window were obtained with the near IR ps laser compared to the ns P2 scribes. In the case of high laser power densities occurring for ultrashort pulses, non linear absorption was probably taking place in the amorphous silicon layer. Therefore, less heating of the rear contact may have occurred with picosecond pulses and could be a reason for the broader process window. An explanation of the cleaner P2 scribe could be that the silicon layer is not completely removed with one single spot (also indicated by the debris in Figure 10), and the subsequent spot cleans the back contact surface by removing the layer residue and/or debris in a second laser irradiation. An example of a clean P2 scribe is shown in Figure 11, where a fluence of $2.76 \mathrm{~J} / \mathrm{cm}^{2}$ and an overlap of $33 \%$ were used.

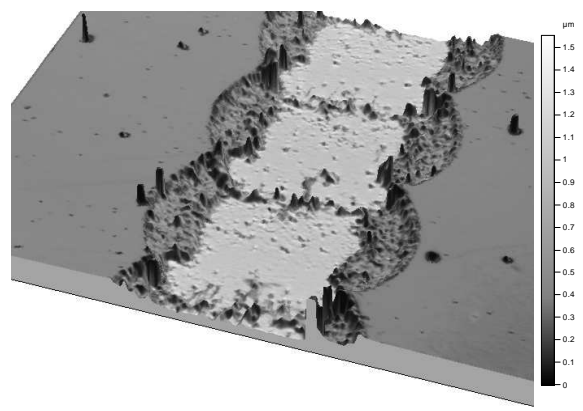

Figure 11: Confocal image of a P2 scribe obtained with a near IR ps laser, at a fluence of $2.76 \mathrm{~J} / \mathrm{cm}^{2}$ and an overlap of $33 \%$.

The same exercise of laser scribing a P2 or a P1 line on a functional solar cell has also been conducted for the ps laser. The I-V curves under illumination before and after the laser step can almost be overlaid, as can be observed in Figure 12.

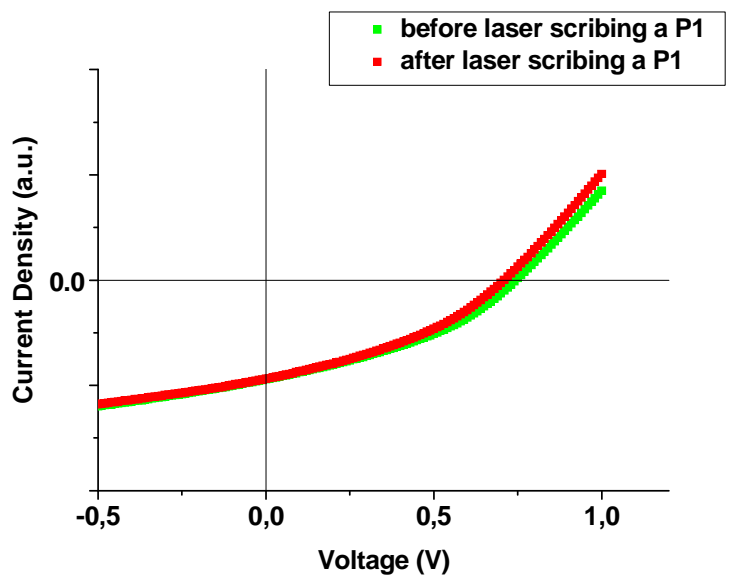

Figure 12: I-V curves under illumination before and after laser scribing a P1 line with the near IR ps laser.

\section{Conclusion}

For the development of thin-film silicon modules on steel foil substrates, depth selective laser scribing is required for the monolithic series interconnection of cells.

This single process step after applying all functional layers of the cell is investigated for near infra-red nanosecond and picosecond lasers. It is observed that for separated ns laser spots, heating of the silver layer from the rear contact might cause an expansion of this layer and from there on, it might push up the silicon and ITO layers. This "P2 spot" ablation mechanism is different from the "P1 spot" ablation process where high vapor pressure is probably breaking and ejecting the $\mathrm{ZnO}$ and $\mathrm{Ag}$ layers.

It is demonstrated that despite a good visual appearance for depth selectivity and a large process window, P1 scribes with near IR nanosecond laser are shunting the solar cell. A possible explanation is that recast of the rear contact were deposited onto the edges of the scribe. These issues with shunting of the PV device have been minimized by scribing with shorter laser pulses, resulting in minor losses in electrical performance after scribing a P1 with a near IR ps laser. Another clear advantage of this laser is the broader process window in depth selectivity for P2 scribes, compared to P2 scribed with the near IR ns laser. This is confirmed by calculations of the peak fluence ablation thresholds of "P2" and "P1" spots with ns and ps lasers. Additionally, a cleaner surface of the back contact in near IR ps P2 scribes is observed, which could be explained by the non linear absorption of ultrashort laser pulses in silicon. 
The comprehension of the ablation mechanisms for P1 and P2 laser scribes is important to understand the possible shunts of thin-film silicon solar cells on steel foil substrates. Further investigations involving laser scribing with other wavelengths and pulse durations are ongoing.

\section{Acknowledgements}

We would like to thank Martijn Tijssen from Delft University of Technology for his contribution in the deposition of nip amorphous silicon solar cells on foil, and Erik Schuring (ECN) for the measurements with the confocal microscope.

\section{References}

[1] Haas, S., Gordijn, A., Stiebig, H. (2008) High Speed Laser Processing for Monolithical Series Connection of Silicon Thin-Film Modules, Prog. Photovolt: Res. Appl. 16, 195-203.

[2] Van Aken, B.B., Dörenkämper, M., Devilee, C., Heijna, M.C.R., Löffler, J. and Soppe, W.J. (2009) Roll to Roll Fabrication Process of Thin-Film Silicon Solar Cells on Steel Foil, Proc. IEEE PVSC Philadelphia.

[3] Löffler, J., Wipliez, L., de Keijzer, M., Bosman, J., Soppe, W. (2009) Depth Selective Laser Scribing of Thin-Films for Roll to Roll Production of Silicon Solar Cells, Proc. ICALEO, Orlando, USA.

[4] Siegman, A.F. (1986) Lasers, University Science Books.

[5] Liu J.M., (1982) Simple technique for measurements of Gaussian-beams spot sizes, Opt. Lett. 7, 196.

[6] Lauzurica, S., Molpeceres, C., García-Ballesteros, J.J., Colina, M., Sánchez-Aniorte, I., Ocaña, J.L., Huber, H., Gandía, J.J. (2009) Comparative study of nanosecond and picosecond laser patterning of thinfilm for photovoltaic solar modules based on a-Si:H, Proc. WLT, Munich.

[7] Hermann, S., Harder, N.-P., Brendel, R., Herzog, D., Haferkamp, H. (2010) Picosecond laser ablation of $\mathrm{SiO}_{2}$ layers on silicon substrates, Appl. Phys. A 99: 151-158.

[8] Hermann, J., Benfarah, M., Bruneau, S., Axente, E., Coustillier, G., Itina, T., Guillemoles, J.-F., Alloncle, P. (2006) Comparative investigation of solar cell thin film processing using nanosecond and femtosecond lasers, J. Phys. D: Appl. Phys. 39 453460.

\section{Meet the author(s)}

Laurie Wipliez has two years of experience in research and development of laser processing of silicon PV materials and devices. She received her Master degree in Materials Science and Nanotechnology from the National Institute of Applied Sciences (INSA) in Rennes (France). She is currently working in the Solar Energy Department at the Energy research Centre of the Netherlands (ECN) on the monolithic series interconnection of thin-film silicon solar cells, and other laser processes for various types of solar cells. 